UC-NRLF

||||||||||||||||||||||||||||||||||||||||

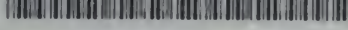

B 3 071 489

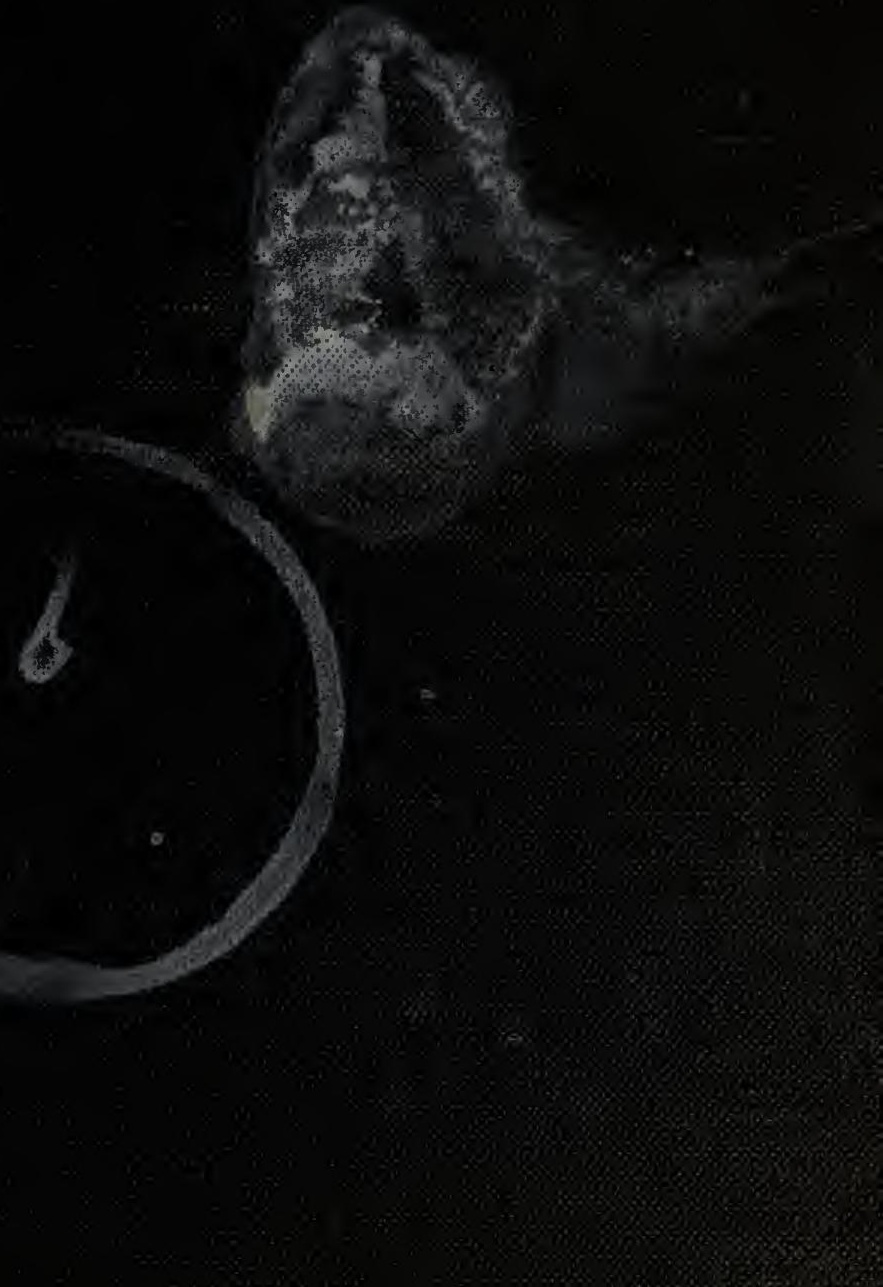




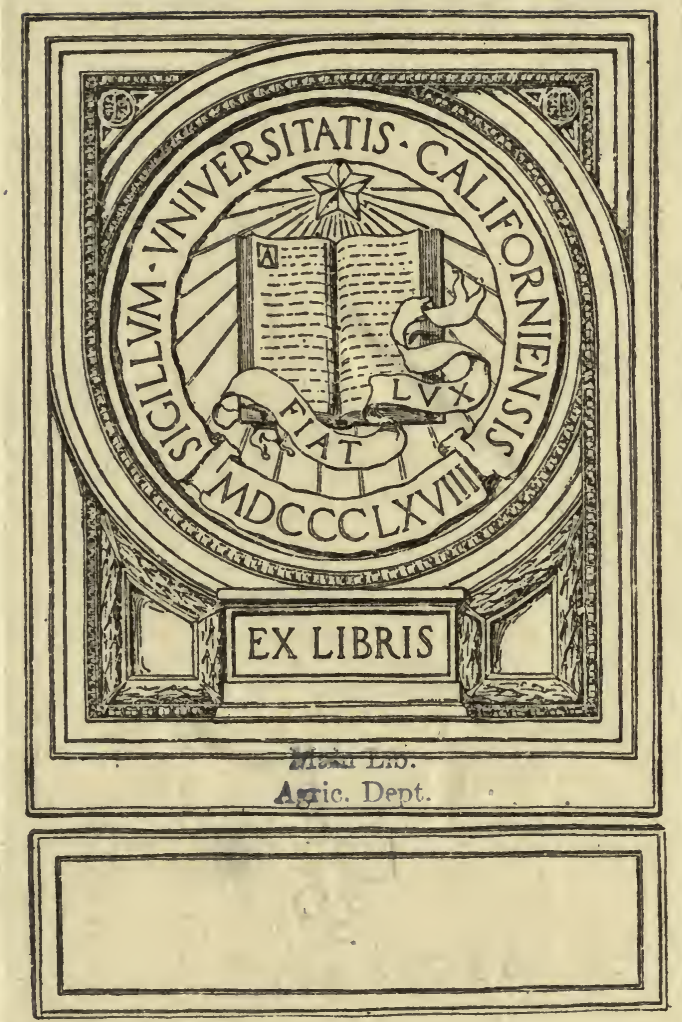


Digitized by the Internet Archive in 2007 with funding from Microsoft Corporation 


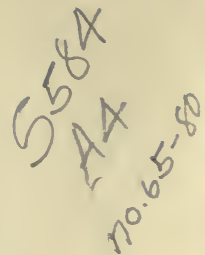

Uom Lib.

Agric. Dent. 
A number of inethóds: finve toen suggested for the estimation of iodin in the presence of other halogens in inorganic soluble compounds. Those involving the conversion of iodid into iodate by potassium permanganate ${ }^{a}$ have been described by Reinige ${ }^{b}$ and by Baubigny and Rivals. ${ }^{c}$ The application of these and of most other methods requires a preliminary destruction of the organic matter, and this, if accomplished in the usual way by fusion with potassium carbonate or a similar alkaline substance, requires much care, and in the case of mixtures containing sulphonated dyes, yields a melt which contains reducing sulphur compounds. These compounds must be removed before the application of Reinige's method, which is also subject to the limitations of volumetric processes in general. With Baubigny's method the distillation requires close attention and much difficulty was experienced in driving off all chlorin without loss of iodin.

Experiments proved that if a solution containing erythrosin is treated with potassium permanganate and nitric acid the organic matter is completely decomposed and chlorin and bromin are converted into volatile products, while all of the iodin remains as iodic acid. The permanganate is converted into a voluminous hydrated oxid of manganese. The chlorin apparently is partly liberated as such and partly converted into an oxygenated compound which is boiled off with difficulty, but may be volatilized in a current of steam or driven off by evaporating the mixture to dryness on a steam bath. When much chlorin is present the last of it can be removed only by treating the residue twice with nitric acid and again evaporating to dryness. That no iodin is lost in this process is shown in Table 1. Iodin may be determined in the residue left after oxidation by the permanganate by taking up in a little water, adding nitric acid, and then dissolving the oxid of manganese in an excess of sulphur dioxid solution, the iodic acid being at the same time reduced. Silver nitrate is added to the clear solution, which is then boiled until the excess of sulphur dioxid is driven off and the silver iodid has coagulated. This is filtered, washed, dried, and weighed in the usual manner.

The experimental results given in Table 1 were obtained on mixtures containing measured amounts of a solution of pure iodin. To prepare this, resublimed iodin was dissolved in a concentrated solution of potassium iodid and a large part of it then precipitated by the addition of water. The precipitated portion was collected on a perforated plate, thoroughly washed with water, and sublimed. The sublimate was dried over calcium chlorid. Portions of this

$a$ Hempel, Ann. Chem. Pharm. 1858, 10\%: 100.

b Zts. anal. chem. 1870, 9: 39.

$c$ Compt. rend. 1903, 13\%: 927. 
iodin were dissolved in a little alkali and diluted to definite volume with water, the solutions so prepared being carefully kept in tightly stoppered flasks and used for the experiments in Table 1. The pipettes and flasks used had been standardized at the United States Bureau of Standards.

TABLE 1.-Estimation of iodin in the presence of chlorin and bromin.

SERIES A. (20 ce of solution $=0.2640$ gram of lodin used.)

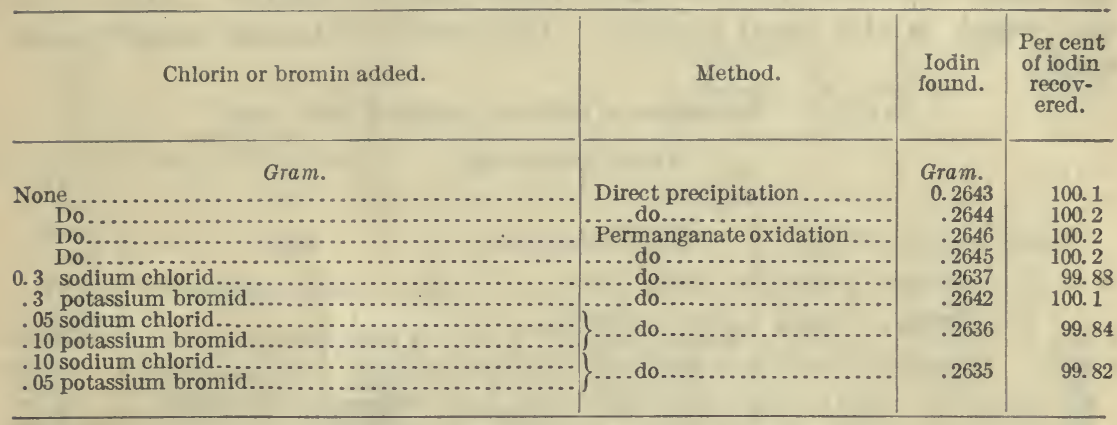

SERIES B. (20 cc of solution $=0.4885$ gram of iodin used.)

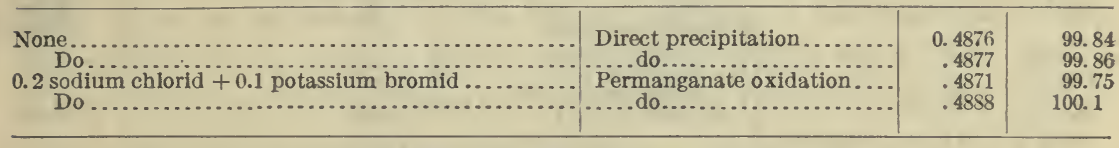

In order to test the applicability of the method to the estimation of iodin in erythrosin a sample of this color was synthesized, the fluorescein, prepared from Kahlbaum's resorcin and phthalic anhydrid, being completely freed from chlorids by repeated precipitation from alkaline solution by acetic acid. This was combined with some of the purified iodin just described, and finally separated as the color acid. This preparation contained no halogen other than iodin, of which the alkaline fusion method indicated 56.40 per cent, as shown in Table 2. After further experimental work on the proper proportion of reagents to be employed the following procedure was adopted:

Place from 0.3 to 0.4 gram of the erythrosin in a porcelain casserol, dissolve this in $5 \mathrm{cc}$ of a 10 per cent sodium hydroxid solution, then add 35 cc of a 7 per cent solution of potassium permanganate. After mixing, cover the vessel with a watch crystal, and add $10 \mathrm{cc}$ of nitric acid, keeping the dish covered. Agitate the mixture, place on a steam bath, and keep covered until spattering ceases, after which remove the watch glass and allow evaporation to proceed to dryness. $^{a}$ Treat the residue with 5 cc of 7 per cent potassium per-

$a$ In the operation of drying particular care should be observed to prevent access of reducing gases to the mixture.

Cir. 65 
manganate and 5 cc of concentrated nitric acid and again evaporate to dryness. Then add about 50 cc of water and 5 ce of concentrated nitric acid to the residue, following this by $40 \mathrm{cc}$ of a saturated solution of sulphur dioxid, and allow the whole to stand with occasional stirring (breaking up the lumps with a glass rod) until the hydrated oxid of manganese has dissolved. Add an excess of silver nitrate to the clear solution, and boil until sulphur dioxid has been expelled and the silver iodid has flocculated. Filter this and weigh in the usual manner. The results obtained are given in Table 2.

TABLE 2.-Estimation of iodin in erythrosin color acid.a

(Theory 60.75 per cent.)

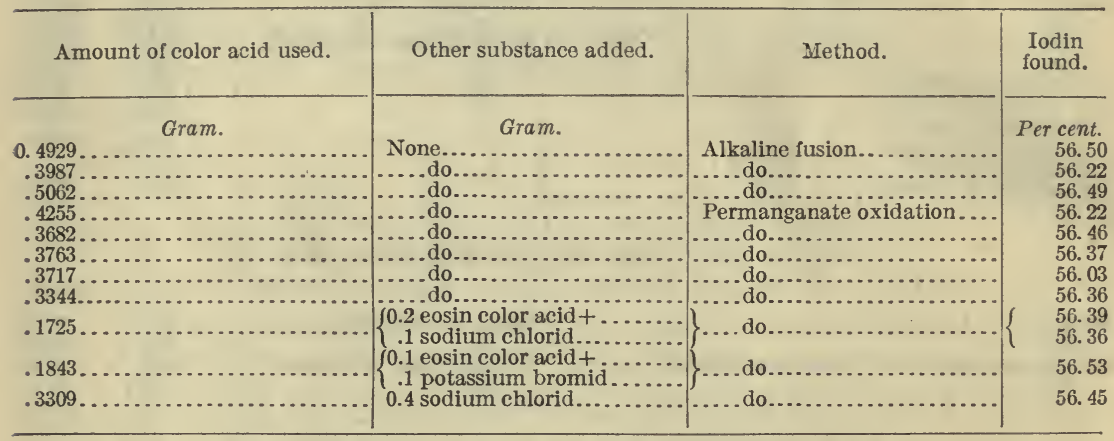

a The erythrosin color acid used in these estimations was air dry.

This procedure was applied to a number of commercial samples containing erythrosin in admixture with ponceau $3 \mathrm{R}$ and with amaranth, and in all cases a complete destruction of the organic matter and closely agreeing results were obtained.

It is believed that this method, with suitable modifications, is applicable to a number of other iodin compounds beside erythrosin, though detailed data substantiating this point have not yet been obtained. Iodin was determined in recrystallized iodoform by first saponifying with alcoholic potash in a pressure flask, evaporating the alcohol, and oxidizing the residue with permanganate as already given. The results are given in Table 3. This procedure may be adopted with the class of compounds that are volatile and easily saponified, but obviously it need only be employed in cases where halogens other than iodin are also present.

Phenyl iodid, as a representative of another water insoluble class, was heated with the oxidizing mixture in an acetylization flask, the reaction mixture being in this case introduced through the tube of the reflux condenser and boiled until the oily drops of phenyl iodid ceased to appear in the condenser, and then for some time longer, rinsing the condenser from time to time with a few cubic centimeters of the 
permanganate solution. The reaction mixture was removed from the flask and evaporated to dryness, proceeding from this point as in the case of erythrosin. These results also are given in Table 3.

TABLE 3.-Estimation of iodin in iodoform and in phenyl iodid.

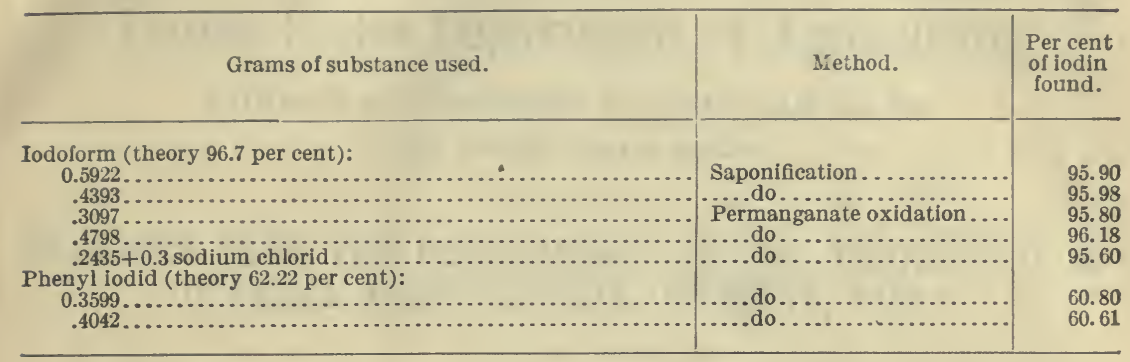

These determinations have not yet been checked against those obtained by other methods, but pending further work to establish the limits of usefulness of the procedure they are reported as found. The method seems to answer perfectly for the estimation of iodin in erythrosin, either when this color alone is present or in a mixture with other colors or with salts of the other halogens. Attention is called particularly to the results given in Table 1, which shows the application of the method in inorganic analysis to the estimation of iodin in the presence of chlorids and bromids. The method seems to possess the double advantage of simplicity and accuracy.

Approved:

\section{James Wilson,}

Secretary of Agriculture.

Washington, D. C., November 5, 1910.

Cir. 65 


\section{RETURN}

\section{MAIN CIRCULATION}

ALL BOOKS ARE SUBJECT TO RECALL RENEW BOOKS BY CALLING $\underline{642-3405}$

\section{DUE AS STAMPED BELOW}

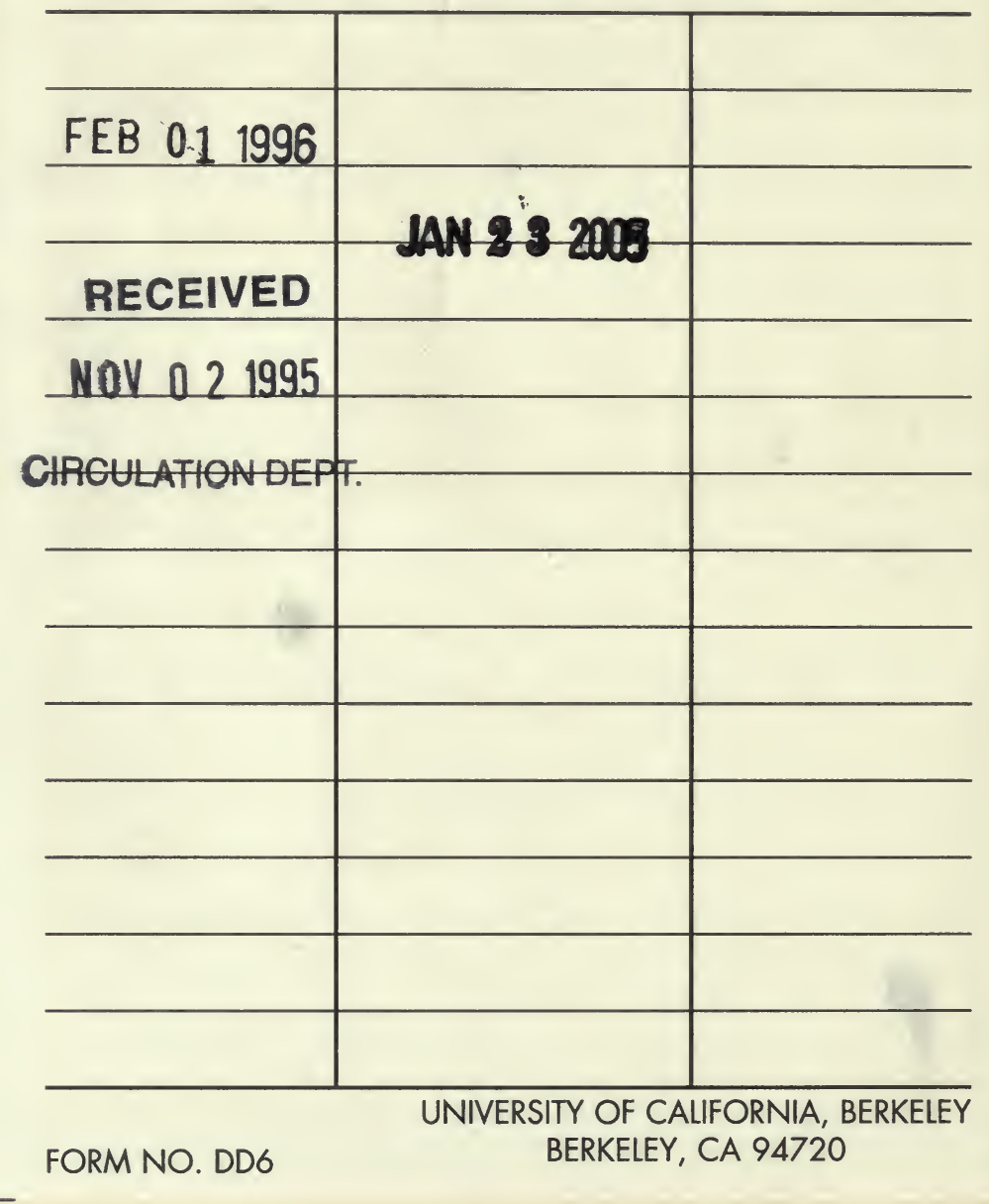


YC 69,40?

$$
\because x y
$$

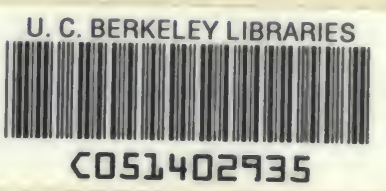


